\title{
Stromal Myofibroblasts Accompany Modifications in the Epithelial Phenotype of Tongue Dysplastic and Malignant Lesions
}

\author{
Marilena Vered • Irit Allon • Amos Buchner • \\ Dan Dayan
}

Received: 30 January 2009 / Accepted: 28 March 2009/Published online: 8 May 2009

(C) The Author(s) 2009. This article is published with open access at Springerlink.com

\begin{abstract}
Stromal myofibroblasts (SMF) associated with various types of carcinomas are believed to emerge under the influence of the tumor cells. Recent studies have shown that SMF may originate from fibroblasts within the tumor stroma or even from carcinoma cells by the process of epithelial-mesenchymal transition. The aim of this study was to investigate the concomitant expression of epithelial membrane antigen and $\alpha$-smooth muscle actin in cells at the tumor-connective tissue interface in human tongue carcinoma, as a possible reflection of epithelialmesenchymal transition. Given its key role in this process, expression of transforming growth factor- $\beta$ in the malignant cells was assessed as well. Immunostaining with $\alpha$ smooth muscle actin was performed on cases of hyperplasia $(n=16)$, mild dysplasia $(n=12)$, moderate-to-severe dysplasia $(n=11)$ and carcinoma $(n=22)$. Transforming growth factor- $\beta$ assessment and double immunostaining with epithelial membrane antigen and $\alpha$-smooth muscle actin were performed only in cases of carcinoma. SMF were significantly associated with carcinomas, while their number in pre-malignant lesions (hyperplasia and dysplasia) was significantly lower $(P<0.001)$. Although SMF were found in all carcinomas, they were heterogeneous in their frequency and patterns of distribution. In addition, $95 \%$ of the carcinomas expressed transforming growth factor- $\beta$ and $41 \%$ exhibited cells positive for both epithelial membrane
\end{abstract}

M. Vered $(\bowtie) \cdot$ I. Allon $\cdot$ A. Buchner $\cdot$ D. Dayan Department of Oral Pathology and Oral Medicine, School of Dental Medicine, Tel Aviv University,

Tel Aviv 69978, Israel

e-mail:1my@netvision.net.il

e-mail: mvered@post.tau.ac.il

M. Vered

Institute of Pathology, Sheba Medical Center,

Tel Hashomer, Israel antigen and $\alpha$-smooth muscle actin. SMF were almost exclusively associated with established carcinomas and not with pre-malignant lesions. Cells that co-expressed epithelial membrane antigen and $\alpha$-smooth muscle actin can be a manifestation of epithelial-mesenchymal transition and, as such, may serve as a source for SMF in these tumors. These findings appear to be linked to the frequent expression of transforming growth factor- $\beta$ by the malignant cells.

Keywords Epithelial-mesenchymal transition - Stromal myofibroblasts $\cdot$ Tongue carcinoma $\cdot$ Transforming growth factor- $\beta$

\section{Introduction}

Oral cancer has consistently ranked among the top ten cancers worldwide with more than 300,000 new cases diagnosed each year $[1,2]$. Despite the recently reported drop in the overall death rate from cancer, the estimated survival rate $(\sim 50 \%)$ and number of deaths from oral cancer remain virtually unchanged [2]. Over $90 \%$ of oral cancers are of the squamous cell carcinoma type.

Solid tumors, such as oral squamous cell carcinoma, have been increasingly perceived as a composite of cancer cells and stromal cells (e.g., fibroblasts, endothelial cells and inflammatory cells) that work in concert towards tumor progression, angiogenesis, local invasion and metastases [3]. It is gradually becoming clearer that of all the stromal cells, the fibroblasts are prominent modifiers of cancer progression $[4,5]$. Our knowledge about these cells is still evolving, but evidence has been accumulating on a subpopulation of fibroblasts, called "activated fibroblasts" with regard to their role in tumor growth and progression $[3,6]$. 
In the early growth stages of epithelial tumors, the neoplasia is embedded in the stroma of a given tissue, which, under the influence of the growth factors secreted by the cancer cells themselves, becomes a "reactive stroma" that is remarkable for its increased number of fibroblasts and enhanced capillary density [3, 7]. Under these conditions, original normal stromal fibroblasts become "activated" and a number of them develop a modified phenotype, similar to that of fibroblasts associated with wound healing, and one which features the expression of $\alpha$ smooth muscle actin. This phenotype is compatible with that of myofibroblasts [8]. The signals that mediate the transition of fibroblasts into stromal myofibroblasts (SMF) are the subject of ongoing investigations. Currently, transforming growth factor- $\beta$ is the leading mediator known to be involved in this transition [9, 10].

In addition to the transition of stromal fibroblasts into SMF, the latter are believed to arise from other origins. Recent studies point to a possible origin from the bone marrow and periadventitial cells (e.g., pericytes and vascular smooth muscle cells) [7]. There is also emerging evidence that the malignant epithelial cells themselves may be a significant source for these cells [11].This phenomenon is termed epithelial-mesenchymal transition during which epithelial cells lose their specific markers and acquire the characteristics of mesenchymal cells [12, 13]. Epithelial-mesenchymal transition, originally described during embryogenesis [12-14], is currently believed to be involved in tumor development and progression [15, 16]. Most notably, down-regulation of epithelial markers (e.g., E (epithelial)-cadherin) concomitant with increased expression of mesenchymal markers (e.g., vimentin) and gain of a fibroblastoid morphology together with an increased invasive potential have been described in oral squamous cell carcinoma cell lines $[17,18]$. Furthermore, down-regulation of E-cadherin expression has been recently associated with poor prognosis in oral squamous cell carcinoma patients [19]. Finally, transforming growth factor- $\beta$ is considered as playing a key role in the epithelial-mesenchymal transition process as well [11-13].

In our previous study using a 4-nitroquinoline 1-oxideinduced rat tongue carcinoma model, we showed that the appearance of SMF was closely associated with the development of carcinoma but not with pre-malignant lesions [20]. Furthermore, on an ultrastructural level, we showed that the carcinoma cells, but not their normal counterparts, acquired cytoplasmic microfilaments that were consistent with contractile microfilaments both in appearance and organization [21]. These events reflect the morphological modifications occurring within the malignant cells, approaching smooth muscle differentiation, probably as part of the epithelial-mesenchymal transition process.
The purpose of the present study was to examine the changes in the occurrence of SMF in tongue epithelial lesions with malignant potential (hyperplasia and dysplasia) and in squamous cell carcinoma, and to assess the expression of transforming growth factor- $\beta$ in cases of carcinoma. In addition, we attempted to identify the presence of carcinoma cells that co-express epithelial membrane antigen and $\alpha$-smooth muscle actin as a reflection of the epithelial-mesenchymal transition process using a double immunostaining method, which was not previously reported in studies on oral cancer in this context.

\section{Materials and Methods}

\section{Study Group}

\section{Study Population}

Records of 22 cases of squamous cell carcinoma of the tongue and 39 cases of premalignant lesions of the tongue consisting of hyperplasia $(\mathrm{N}=16)$, mild dysplasia $(\mathrm{N}=12)$, and moderate-to-severe dysplasia $(\mathrm{N}=11)$ were retrieved from the files of the Department of Oral Pathology, School of Dental Medicine, Tel-Aviv University and Institute of Pathology, The Chaim Sheba Medical Center, Tel Hashomer. Diagnoses were reevaluated and classified by two oral pathologists (MV and DD) according to the World Health Organization classification of head and neck tumors [22]. This study was approved by the Helsinki committee of the Sheba Medical Center.

\section{Immunohistochemical Stains}

Three $\mu$ wide sections had been cut from the 61 blocks containing the biopsy specimens of the study cases. They were mounted on positive-charged microscope slides (Optiplus $^{\mathrm{TM}}$, Biogenex, San Ramon, CA, USA), dewaxed in xylene, dehydrated in ethanol, rinsed in distilled water, placed in $3 \% \mathrm{H}_{2} \mathrm{O}_{2}$, and rinsed again in distilled water. The slides were placed in citrate buffer solution, $\mathrm{pH}=6$, in a microwave oven at $92^{\circ} \mathrm{C}$ for $10 \mathrm{~min}$ for retrieval of antigens. After cooling, the slides were exposed to the primary $\alpha$-smooth muscle actin antigen (clone 1A4, 1:100, Dako A/S, Denmark) for $60 \mathrm{~min}$ at room temperature. They were then rinsed in phosphate buffered saline (PBS). The universal immune peroxidase polymer anti-mouse rabbit Histofine $^{\circledR}$ (Multi) kit (Nichirei, Tokyo, Japan) was used for the detection of antibodies. The sections were rinsed in PBS, reacted with an amino ethyl-carbazole (AEC) substrate chromogen kit (Zymed, San Francisco, CA, USA), rinsed in PBS, counterstained in Mayer's hematoxylin (Pioneer Research Chemicals, Colchester, UK) and covered 
with glycerol vinyl alcohol (GVA) mounting medium (Zymed, San Francisco, CA, USA). Positive control tissues comprised of bowel wall for $\alpha$-smooth muscle actin, breast for epithelial membrane antigen and placenta for transforming growth factor- $\beta$. Negative controls were achieved by performing the staining procedures with omission of the primary antibody.

Only the squamous cell carcinoma sections were submitted to additional immunostaining by transforming growth factor- $\beta$ (1:25, LabVision, Fremont, CA, USA) and double staining with $\alpha$-smooth muscle actin and epithelial membrane antigen (clone ZCE 113, 1:50, Zymed, San Francisco, CA, USA), employing a double chromogen reaction, where the former was visualized by 3,3'-diaminobenzidine (DAB) and the latter by Fast-Red (Biocare, Concord, CA, USA). Epithelial membrane antigen was chosen as a marker for epithelial differentiation [23] using a typical membranous cellular localization to discriminate it from cytoplasmic $\alpha$-smooth muscle actin positivity.

Immunomorphometric Assessment of the $\alpha$-Smooth Muscle Actin-Stained SMF

The method employed in the present study was used by us previously [20]. In brief, a 100-square grid (Olympus, Tokyo, Japan) was mounted on the microscope. Each crossing between a horizontal and vertical line was termed as an "intersection". At x400 magnification, the grid was located on the left border of the tissue, immediately beneath the epithelium, where its upper border tangentially touched the tip of the adjacent epithelial rete ridges. The $\alpha$-smooth muscle actin-stained cells, compatible with myofibroblasts, were counted within the connective tissue covered by the 3 rows of the grid (30 squares, 44 intersections) closest to the epithelium. According to the point-counting method, the $\alpha$ smooth muscle actin-stained cells that overlapped an intersection in the established area were counted, excluding all positively stained cells in the blood vessel walls. When counting of the first field was completed, the grid was moved to the next field, using the peripheral border of the grid as the reference point. A total of 10 representative fields were counted in each case. For areas containing carcinoma, the fields were counted at the periphery of the tumor islands at the invasive front. The number of $\alpha$ smooth muscle actin-stained cells overlapping intersections in the 10 fields in each case was summed up and expressed as the percentage of the total number of intersections (i.e., 440). The results were expressed as the mean percentage of $\alpha$-smooth muscle actin-stained cells per intersection in each study group. For example, the mean percentage of $\alpha$ smooth muscle actin-stained cells per intersection in the 22 cases of the squamous cell carcinoma group was calculated as follows: all $\alpha$-smooth muscle actin-positive intersections in 10 fields were summed up and divided by 440 . The results of all these 22 cases were added together, divided by 22 and multiplied by 100 .

Pattern of Distribution of the SMF in Cases of Squamous Cell Carcinoma

The immunohistochemically stained squamous cell carcinoma slides were examined for the pattern of distribution of the SMF. The cases were classified according to two dominant patterns: "spindle" and "network". In the "spindle" pattern, visualization at low and medium power revealed stromal $\alpha$-smooth muscle actin-stained myofibroblasts with a spindle-shape morphology tightly adhering to the periphery of the carcinoma islands/nests in one-to-three concentric layers. In the "network" pattern, SMF were exceptionally abundant and had a plump appearance, and their proportion occasionally exceeded that of the carcinomatous component. They were organized in short-to medium-length intersecting bundles and, at a higher magnification, their high density gave the impression of multilayering, thus the term "network".

Staining Pattern of Transforming Growth Factor- $\beta$ in Squamous Cell Carcinoma

Expression of transforming growth factor- $\beta$ was assessed semi-quantitatively, where positive cases were defined as those with more than $10 \%$ of SCC cells exhibiting transforming growth factor- $\beta$ reactivity [24]. Cytoplasmic and/ or membranous transforming growth factor- $\beta$ staining was counted. There were two distinct staining patterns among the positive cases: one was a "diffuse pattern" in which most of the carcinoma cells were transforming growth factor- $\beta$ positive and the other was a "focal pattern" in which positive cells were irregularly distributed and displayed mixed negative and positive areas.

Assessment of the Carcinoma Cells Co-Expressing Epithelial Membrane Antigen and $\alpha$-Smooth Muscle Actin

Expression of positive epithelial membrane antigen immunoreactivity consisted of purple membranous and occasionally cytoplasmic staining while that of $\alpha$-smooth muscle actin consisted of brown cytoplasmic staining. Each section from the carcinoma group was thoroughly examined at $\times 400$ with special emphasis on the tumor-connective tissue interface and invasion front for identification of cells that were simultaneously immunolabeled for both stains. Cases were assessed qualitatively and assigned as "positive" if carcinoma cells with these characteristics were found in the entire section. The double-stained carcinoma cells often appeared in small islands, clusters or even as isolated cells. 
Table 1 Mean $\%$ of $\alpha$-smooth muscle actin-positive SMF/intersection according to study groups

${ }^{*} P<0.001$
Study group

Mean \pm SD $\%$ of stained SMF (range)

Hyperplasia

$0.9 \pm 0.5(0.2-2.6)$

Mild dysplasia

$1.1 \pm 0.5(0.5-2.0)$

Moderate-to-severe dysplasia

$0.8 \pm 0.3(0.3-1.3)$

Squamous cell carcinoma

\section{Data Analysis}

Differences in the mean percentage of $\alpha$-smooth muscle actin-positive myofibroblasts per intersection among the study groups were analyzed using analysis of variance (ANOVA) with a log transformation test. Statistical significance was set at $P<0.05$. The Statistical Package for the Social Sciences (SPSS 14, Chicago, IL, USA) software was used for computations.

\section{Results}

Mean $\%$ of $\alpha$-Smooth Muscle Actin-Positive SMF Per Intersection According to Study Groups

The results are summarized in Table 1. SMF were infrequent in cases of premalignant lesions (hyperplasia and dysplasia) irrespective of the severity of morphological and cytological changes. The mean percent of SMF in these
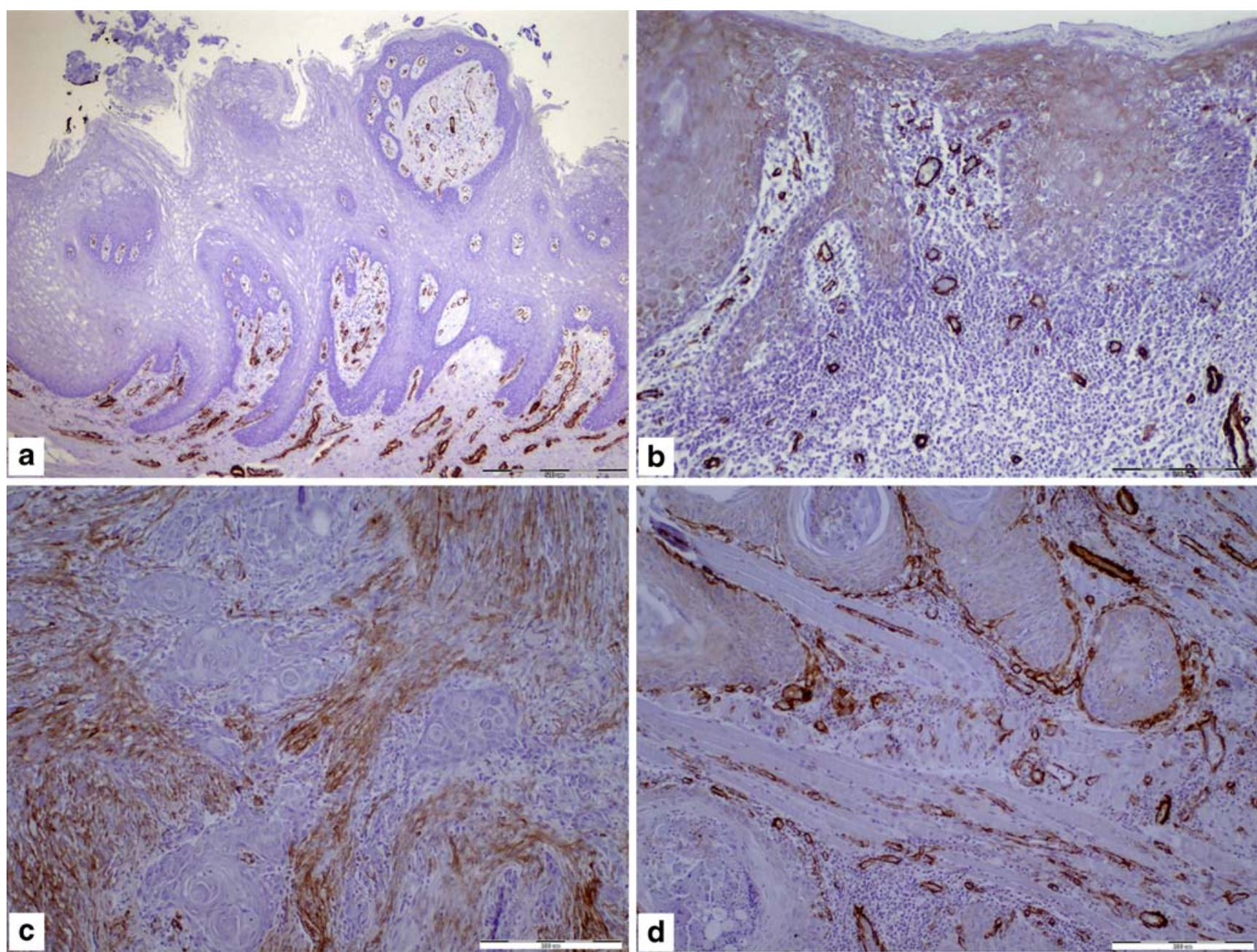

Fig. 1 a Epithelial hyperplasia and $\mathbf{b}$ moderate-to-severe dysplasia showing $\alpha$-smooth muscle actin immunostaining only in smooth muscle cells within blood vessel walls. No $\alpha$-smooth muscle actin immunostaining corresponding to stromal myofibroblasts could be

identified. c Abundance of stromal myofibroblasts in a "network" and, d "spindle" pattern (anti- $\alpha$-smooth muscle actin antibody, amino ethyl-carbazole (AEC) method; a-bar $500 \mu$; b, c, d—bar $200 \mu$ ) 
cases was about $1 \%$, and no significant differences were found among these groups $(P>0.05)$. In contrast, there was a sharp increase in the mean percent of the SMF in the carcinoma group $(14.7 \pm 12.8 \%)$. The difference between the malignant and premalignant groups was highly significant $(P<0.001)$.

\section{$\alpha$-Smooth Muscle Actin-Positive SMF Staining Patterns}

Immunomorphometric measurements revealed that $\alpha$ smooth muscle actin-positive SMF were scarce in cases of hyperplasia and dysplasia, irrespective of the severity of the latter (Fig. 1a and b). The appearance of SMF in remarkable numbers was associated with evidence of malignancy. Even among cases of carcinoma, however, the frequency of these cells was not uniform, ranging from cases with few SMF to cases in which SMF constituted a major component of all the stroma (Table 2). Five (23\%)

Table 2 Cases of tongue squamous cell carcinoma according to increasing values of mean $\%$ of SMF and the respective pattern of distribution of SMF, epithelial membrane antigen (EMA) and $\alpha$ smooth muscle actin (SMA) double staining and transforming growth factor (TGF)- $\beta$ pattern

\begin{tabular}{|c|c|c|c|c|}
\hline $\begin{array}{l}\text { Case } \\
\text { no. }\end{array}$ & $\begin{array}{l}\text { Mean \% } \\
\text { of SMF }\end{array}$ & $\begin{array}{l}\text { Pattern of } \\
\text { distribution of } \\
\text { SMF }\end{array}$ & $\begin{array}{l}\text { EMA and } \alpha- \\
\text { SMA double } \\
\text { staining }\end{array}$ & $\begin{array}{l}\text { TGF- } \beta \\
\text { pattern }\end{array}$ \\
\hline 1 & 1.2 & Spindle & + & Focal \\
\hline 2 & 1.3 & Spindle & + & Focal \\
\hline 3 & 1.8 & Spindle & - & Focal \\
\hline 4 & 3.7 & Spindle & - & Focal \\
\hline 5 & 4.1 & Spindle & - & Focal \\
\hline 6 & 7 & Spindle & - & Focal \\
\hline 7 & 7.2 & Spindle & - & Focal \\
\hline 8 & 7.8 & Spindle & - & Diffuse \\
\hline 9 & 8.75 & Spindle & - & Diffuse \\
\hline 10 & 10.7 & Spindle & + & Focal \\
\hline 11 & 11 & Spindle & - & $\begin{array}{l}\text { No } \\
\text { Stain }\end{array}$ \\
\hline 12 & 11.5 & Spindle & - & Focal \\
\hline 13 & 12.65 & Spindle & - & Focal \\
\hline 14 & 13 & Spindle & - & Diffuse \\
\hline 15 & 13.5 & Spindle & + & Diffuse \\
\hline 16 & 16.1 & Spindle & - & Focal \\
\hline 17 & 24.2 & Spindle & - & Diffuse \\
\hline 18 & 24.4 & Network & + & Focal \\
\hline 19 & 28.2 & Network & + & Focal \\
\hline 20 & 28.5 & Network & + & Diffuse \\
\hline 21 & 33.6 & Network & + & Diffuse \\
\hline 22 & 51.4 & Network & + & Focal \\
\hline
\end{tabular}

$(+)$, positive double immunostaining and $(-)$ negative double immunostaining cases of carcinoma exhibited a "network" pattern of SMF with large, usually vesicular nuclei with abundant cytoplasm that demonstrated cytoplasmic projections, which interconnected among neighboring SMF and formed a network around the carcinoma islands (Fig. 1c). The fine boundary between the stromal and epithelial compartments was often breached and a physical connection between the SMF and the carcinoma cells was apparent. Under these circumstances, the SMF acquired an epithelioid appearance, forming syncytial connections between them and the carcinoma cells. The "network" pattern could be seen throughout the tumor stroma and was not pronounced at the invasion front. The "spindle" pattern was observed in 17 (77\%) cases. The SMF were aligned in an orderly manner at the periphery of the tumor islands/nests and there were distinct borders between these cells and the malignant ones (Fig. 1d).

Transforming Growth Factor- $\beta$ Staining Pattern in Cases of Squamous Cell Carcinoma

Transforming growth factor- $\beta$ positivity was found in $95 \%$ of the carcinomas, out of which $63 \%$ had a "focal" and $32 \%$ had a "diffuse" staining pattern (Table 2, Fig. 2a and b, respectively). The depth of the tumor and the area of the invasion front were usually remarkable for the concentration of transforming growth factor- $\beta$-positive carcinoma cells, even in the "focal" cases. The "diffuse" pattern of transforming growth factor- $\beta$ staining became obvious in cases with a mean percent of SMF of $\sim 8 \%$ and higher.

Double Epithelial Membrane Antigen and $\alpha$-Smooth Muscle Actin Immunostaining in Cases of Squamous Cell Carcinoma

Nine $(41 \%)$ cases of carcinoma that had been submitted to double immunostaining procedures were designated as "positive" since they exhibited cells that co-expressed epithelial membrane antigen and $\alpha$-smooth muscle actin (Table 2). Typical epithelial membrane antigen staining was usually retained in well-differentiated areas, where it was visualized as a purple, continuous, and slightly granular membranous stain that highlighted the intercellular regions. In these areas, the cytoplasm of the carcinoma cells often had a light purplish-to-pink color. In other less differentiated areas, membranous epithelial membrane antigen reactivity was reduced and appeared as an interrupted band with occasional very pale cytoplasmic stain. A pattern of progressing loss of membranous epithelial membrane antigen staining was seen at the periphery of the tumor islands or in small clusters situated within the depth of the sections and at the invasive front. The cytoplasm of these cells was light brown and they were barely distinguishable 

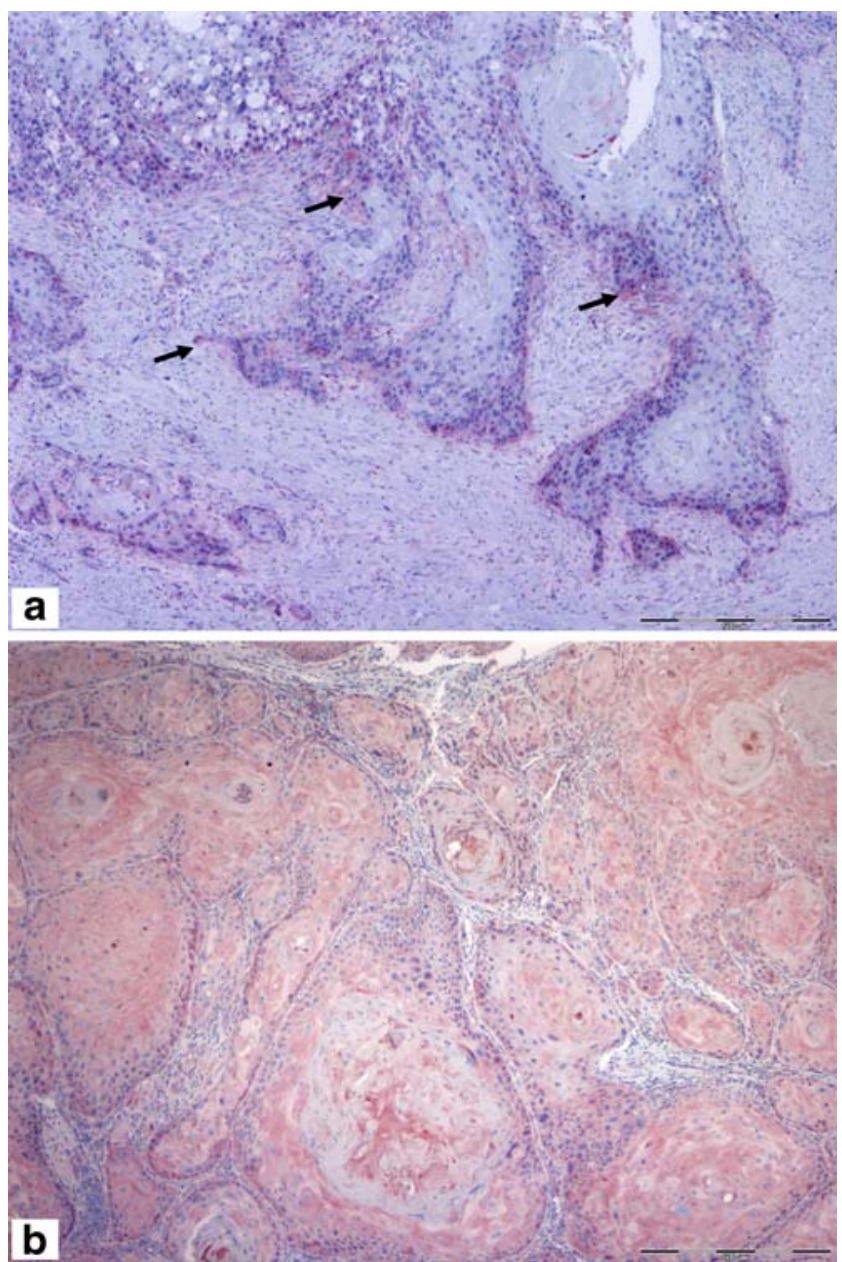

Fig. 2 a Transforming growth factor- $\beta$ positivity in carcinoma cells in a "focal" pattern as indicated by arrows; $\mathbf{b}$ a "diffuse" pattern (antitransforming growth factor- $\beta$ antibody, amino ethyl-carbazole (AEC) method; bar $500 \mu$ )

from the surrounding mesenchymal cells on the basis of nuclear pleomorphism and hyperchromatism or according to their connection to a cluster of carcinoma cells that still maintained some of the epithelial membrane antigen immunoreactivity. These cells occasionally displayed a stellate-shaped or fusiform architecture with cytoplasmic projections, thereby assuming a mesenchymal appearance (Fig. 3a). In these areas, some of the carcinoma cellsespecially those presenting morphological alterationsco-expressed epithelial membrane antigen and $\alpha$-smooth muscle actin. The former stain had a purple membranous or cytoplasmic appearance and the latter stain was brown and cytoplasmic (Fig. 3a and b).

The higher the SMF counts, the more frequent the "network" distribution of the SMF tended to be, and the more frequent the presence of carcinoma cells coexpressing epithelial membrane antigen and $\alpha$-smooth muscle actin (Table 2).

\section{Discussion}

The results of our study showed that presence of SMF was associated with carcinoma of the tongue, while these cells were sparse to absent in pre-malignant lesions. In addition, we found that tumors were heterogeneous in the extent of SMF and their pattern of distribution and morphological features. Indications of an association between squamous cell carcinoma and SMF were reported in previous studies that employed cell lines [25] and specimens of squamous
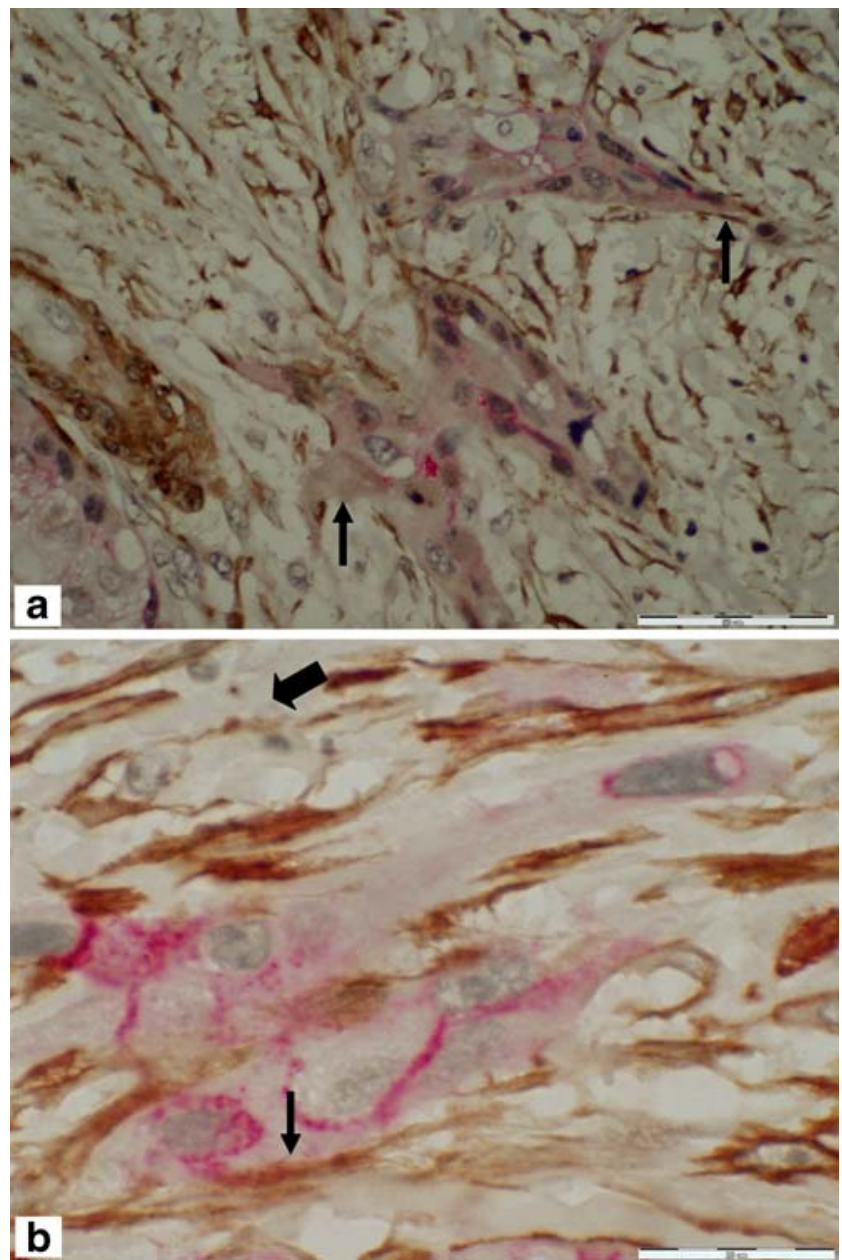

Fig. 3 a Small islands of carcinoma cells at the invading front showing a stellate-shaped and fusiform architecture with cytoplasmic projections, some with a fibroblastoid appearance. Remnants of epithelial membrane antigen staining (purple membranous/cytoplasmic stain) disclose their epithelial origin. Brownish cytoplasmic staining of $\alpha$-smooth muscle actin is observed in a few foci within the carcinoma cells (arrows) (anti-epithelial membrane antigen and anti- $\alpha$-smooth muscle actin antigen antibodies, Fast-red and 3,3'diaminobenzidine (DAB) double immunostaining; bar $50 \mu$ ). b Spindle carcinoma cells with remnants of epithelial membrane antigen staining and an area of brown cytoplasmic stain compatible with $\alpha$ smooth muscle actin positivity (thin arrow). A cluster of carcinoma cells negative for epithelial membrane antigen and positive for $\alpha$ smooth muscle actin is seen in the upper left corner (thick arrow) (bar $20 \mu)$ 
cell carcinoma of the entire oral cavity [26, 27]. The presence of SMF was recently analyzed in a series of tongue carcinomas versus normal and dysplastic epithelial lesions from the entire oral mucosa [28]. In that study, in which frequency of SMF was assessed by a vague semiquantitative scale, it was reported that SMF were found exclusively in carcinoma $(\sim 60 \%)$ and not in any of the dysplastic lesions. In contrast, in the present study, tongue carcinomas were analyzed versus tongue dysplastic lesions and the frequency of SMF was assessed by a systematic immunomorphometric method. Accordingly, we found SMF in all the cases of carcinoma and small numbers of SMF were also identified in the dysplastic lesions.

Although we investigated a relatively small number of tumors, not all the examined cases presented a homogeneous pattern of SMF. The proportion between an existing malignancy and SMF ranged from tumors in which the SMF were incidental to tumors in which they predominated. Furthermore, the layout of the SMF around the tumor islands generated different patterns, such that tumors with fewer SMF usually displayed spindle, delicate SMF organized in bundles that subtly surrounded the carcinoma at its periphery, while tumors with an abundance of SMF often featured epithelioid SMF that amalgamated with the carcinoma cells and were organized in a syncytium-like, cellular network. These differences might have an impact on defining the biological aggressiveness of the tumors, based on the fact that the SMF are considered as the biological "factories" for a vast range of mediators that back up, enhance and promote tumor's invasion. These mesenchymal cells are major suppliers of matrix metalloproteinases, whose function has been recently extended and consists not only of degradation of extra-cellular matrix proteins but also of an active part in tumor initiation, growth, migration, invasion, formation of metastasis, angiogenesis and selection of apoptosis-resistant clones [29]. An association between metalloproteinases and a more aggressive biological behavior of oral squamous cell carcinoma and a poorer prognosis has been reported [30, 31].

We also observed a trend wherein the more frequent the expression of cancer-derived transforming growth factor- $\beta$, the more abundant were the SMF in the adjacent tumor. This is in accordance with the recognized key role of this growth factor in the transformation of resident fibroblasts into myofibroblasts $[9,10]$. In addition, it is known that transforming growth factor- $\beta$ plays a crucial role, together with other factors, in another biological process - epithelialmesenchymal transition, which has been described as a physiological process during normal embryogenesis on the one hand, and in pathological conditions, such as fibrosis and cancer, on the other hand $[12,13,32]$. In the present study, some of the SMF had an epithelioid appearance at the tumor-connective tissue interface, while some of the carcinoma cells demonstrated a spindle, fibroblastoid appearance due to a nearly total loss of cohesion with their neighboring cells. These morphological features highlighted the blurred boundary between the epithelial and mesenchymal phenotypes. In addition, double immunoreactivity revealed that malignant cells were more commonly found in tumors that displayed high numbers of SMF with a "network" pattern of distribution. It seems that under certain conditions determined by the tumor needs, the reservoir of SMF (mostly of resident fibroblast origin) is probably enriched by carcinoma cells that could undergo epithelial-mesenchymal transition [12, 13, 32, 33]. These observations can be explained, in part, in terms of the autocrine effect of cancer-derived transforming growth factor- $\beta$. This growth factor interferes with the essential intercellular epithelial junctional complexes of epithelial (E)-cadherin and $\beta$-catenin, whereby E-cadherin-mediated sequestration of $\beta$-catenin at the cell membrane is abolished. As a result, $\beta$-catenin localizes to the nucleus and subsequently activates transcriptional factors, such as Snail, which will ultimately down-regulate E-cadherin expression and lead to loss of intercellular cohesion [32, 33]. On clinical grounds, reduced expression of E-cadherin in oral carcinomas has been consistently found to be associated with an invasive growth pattern and a shortened 5 -year survival [19, 34]. In tongue carcinoma, in particular, low expression of E-cadherin was found to be predictive for cervical lymph node metastases [35].

Our positive double immunostaining results revealed a continuum of cells with undistinguishable intercellular borders, ranging from unmistakably epithelial membrane antigen-positive carcinoma cells to weakly-to-no epithelial membrane antigen staining, further to both epithelial membrane antigen - and $\alpha$-smooth muscle actin - stained carcinoma cells, and finally, to strongly $\alpha$-smooth muscle actin-stained SMF. This is the first study on human oral carcinoma that used a double immunostaining method to show progressive reduction in the expression of epithelial membrane antigen with concomitant gain of $\alpha$-smooth muscle actin. These changes reflect one aspect of the plasticity in the phenotype of the malignant epithelial cells, as long as it serves the aim of facilitating local invasion and metastatic dissemination $[11,16]$. In a previous study in an animal model of tongue carcinoma, we showed at an ultrastructural level that neoplastic cells at the tumor-connective tissue interface acquired morphologic modifications approaching smooth muscle differentiation by developing a cytoplasmic system of contractile microfilaments, probably as part of the epithelial-mesenchymal transition process [21].

Epithelial membrane antigen was used in this study as a structural marker for epithelial differentiation [23]. Other 
studies on epithelial-mesenchymal transition used Ecadherin as a functional marker for epithelial intercellular junctional complexes and showed its down-regulation as a reflection of underlying epithelial-mesenchymal transition, principally mediated by transforming growth factor- $\beta[12$, 32]. Although epithelial membrane antigen and E-cadherin belong to different classes of molecules with various functions, a recent study on breast cancer showed restricted expression of both molecules during the epithelialmesenchymal transition process [36].

In summary, the present study was the first to use a double immunohistochemical technique in human tongue carcinoma in order to investigate the possibility of an epithelial-mesenchymal transition process. It has been shown that SMF, which were almost exclusively associated with established tongue carcinoma, emerged concomitant with morphological and immunohistochemical modifications in the carcinoma cells. Transforming growth factor- $\beta$ apparently plays a role in both the emergence of SMF and in the changes in the malignant cells. This is supported by the observed trend of its higher expression in cases with abundant SMF and frequent tumor cells co-expressing epithelial membrane antigen and $\alpha$-smooth muscle actin. The present results justify investigations on a larger scale to assess whether the frequency of the carcinoma cells undergoing such modifications may be correlated with variations in the biological behavior of oral squamous cell carcinoma and clinical outcomes [37]. Realizing that the SMF are part of the tumor that contribute to its progression and that the malignant cells are in a dynamic state of changing phenotypes toward a mesenchymal differentiation could help explain the partial response to routine anticancer treatment approaches as is often seen in oral squamous cell carcinoma, implying that future cancer therapies would have to target stromal constituents and should not focus solely on "conventional" cancer cells.

Acknowledgements The authors would like to thank Mrs. Hana Vered for technical assistance and Mrs. Esther Eshkol for editorial assistance. The study was supported by the Vladimir Schreiber Research Fund and the Tibor Bilha and Elizabeth Rubinstein De Bilha Research Fund, Sackler Faculty of Medicine, Tel Aviv University.

Open Access This article is distributed under the terms of the Creative Commons Attribution Noncommercial License which permits any noncommercial use, distribution, and reproduction in any medium, provided the original author(s) and source are credited.

\section{References}

1. Kademani D (2007) Oral Cancer. Mayo Clin Proc 82(7):878-887 (erratum: Mayo Clin Proc 2007 82(8):1017)
2. Choi S, Myers JN (2008) Molecular pathogenesis of oral squamous cell carcinoma: implications for therapy. J Dent Res 87(1):14-32

3. Kalluri R, Zeisberg M (2006) Fibroblasts in cancer. Nat Rev Cancer 6(5):392-401

4. Tlsty TD, Hein PW (2001) Know thy neighbor: stromal cells can contribute oncogenic signals. Curr Opin Genet Dev 11(1):54-59

5. Elenbaas B, Weinberg RA (2001) Heterotopic signaling between epithelial tumor cells and fibroblasts in carcinoma formation. Exp Cell Res 264(1):169-184

6. Mueller MM, Fusening NE (2004) Friends or foes-bipolar effects of the tumor stroma in cancer. Nat Rev Cancer 4(11):839-849

7. Zeisberg EM, Potenta S, Xie L et al (2007) Discovery of endothelial to mesenchymal transition as a source for carcinomaassociated fibroblasts. Cancer Res 67(21):10123-10128

8. Tomasek JJ, Gabbiani G, Hinz B et al (2002) Myofibroblasts and mechano-regulation of connective tissue remodeling. Nat Rev Mol Cell Biol 3(5):349-363

9. Lohr M, Schimdt C, Ringel J et al (2001) Transforming growth factor $\beta-1$ induces desmoplasia in an experimental model of human pancreatic carcinoma. Cancer Res 61(2):550-555

10. Aoyagi Y, Oda T, Kinoshita $T$ et al (2004) Overexpression of TGF- $\beta$ by infiltrated granulocytes correlates with the expression of collagen mRNA in pancreatic cells. Br J Cancer 91(7):13161326

11. De Wever O, Mareel M (2003) Role of tissue stroma in cancer cell invasion. J Pathol 200(4):429-447

12. Thiery JP, Sleeman JP (2006) Complex networks orchestrate epithelial-mesenchymal transitions. Nat Rev Mol Cell Biol 7 (2):131-142

13. Nawshad A, LaGamba D, Polad A et al (2005) Transforming growth factor- $\beta$ signaling during epithelial-mesenchymal transformation: implications for embryogenesis and tumor metastasis. Cells Tissues Organs 179(1-2):11-23

14. Trelstad RL, Hay ED, Revel JD (1967) Cell contact during early morphogenesis in the chick embryo. Dev Biol 16(1):78-106

15. Yang J, Mani SA, Donaher JL et al (2004) Twist, a master regulator of morphogenesis, plays an essential role in tumor metastasis. Cell 117(7):927-939

16. Radisky DC, Kenny PA, Bissell MJ (2007) Fibrosis and cancer: do myofibroblasts come also from epithelial cells via EMT? J Cell Biochem 101(4):830-839

17. Takkunen M, Grenman R, Hakkunen M et al (2006) Snaildependent and-independent epithelial-mesenchymal transition in oral squamous carcinoma cells. J Histochem Cytochem 54 (11): $1263-1275$

18. Yokoyama K, Kamata N, Hayashi E et al (2001) Reverse correlation of E-cadherin and snail expression in oral squamous cell carcinoma in vitro. Oral Oncol 37(1):65-71

19. Diniz-Freitas M, Garcia-Caballero T, Antunez-Lopez J et al (2006) Reduced E-cadherin is an indicator of unfavorable prognosis in oral squamous cell carcinoma. Oral Oncol 42(2):190-200

20. Vered M, Allon I, Buchner A et al (2007) Stromal myofibroblasts and malignant transformation in a $4 \mathrm{NQO}$ rat tongue carcinogenesis model. Oral Oncol 43(10):999-1006

21. Vered M, Polak-Charcon S, Babushkin T et al (2008) 4NQOinduced tongue carcinoma: an ultrastructural study. Ultrastruct Pathol 32(5):199-205

22. Gale N, Pilch BZ, Sidransky D et al (2005) Epithelial precursor lesions. In: Barnes L, Eveson JW, Reichart P et al (eds) WHO classification of tumours. Pathology and genetics. Head and neck tumours. IARC, Lyon, pp 177-179

23. Pinkus GS, Kurtin PJ (1985) Epithelial membrane antigen-a diagnostic discriminant in surgical pathology: immunohistochemical profile in epithelial, mesenchymal, and hematopoietic 
neoplasms using paraffin sections and monoclonal antibodies. Hum Pathol 16(9):929-940

24. Logullo AF, Nonogaki S, Miguel RE et al (2003) Transforming growth factor betal (TGFbeta1) expression in head and neck squamous cell carcinoma patients as related to prognosis. J Oral Pathol Med 32(3):139-145

25. Lewis MP, Lygoe KA, Nystrom ML et al (2004) Tumour-derived TGF-betal modulates myofibroblast differentiation and promotes HGF/SF-dependent invasion of squamous carcinoma cells. Br J Cancer 90(4):822-832

26. Barth PJ, Schenck zu Schweinsberg T, Ramaswamy A et al (2004) CD34+ fibrocytes, alpha-smooth muscle antigen-positive myofibroblasts, and CD117 expression in the stroma of invasive squamous cell carcinomas of the oral cavity, pharynx, and larynx. Virchows Arch 444(3):231-234

27. Vered M, Shohat I, Buchner A et al (2005) Myofibroblasts in stroma of odontogenic cysts and tumors can contribute to variations in the biological behavior of lesions. Oral Oncol 41 (10):1028-1033

28. Kellermann MG, Sobral LM, da Silva SD et al (2007) Myofibroblasts in the stroma of oral squamous cell carcinoma are associated with poor prognosis. Histopathology 51(6):849-852

29. Lynch CC, Matrisian LM (2002) Matrix metalloproteinases in tumor-host cell communication. Differentiation 70(9-10):561573
30. Patel PB, Shah PM, Rawal UM et al (2005) Activation of MMP-2 and MMP-9 in patients with oral squamous cell carcinoma. J Surg Oncol 90(2):81-88

31. de Vicente GJ, Fresno MF, Villalain L et al (2005) Expression and clinical significance of matrix metalloproteinase-2 and matrix metalloproteinase-9 in oral squamous cell carcinoma. Oral Oncol 41(3):283-293

32. Prime SS, Davies M, Pring M et al (2004) The role of TGF- $\beta$ I epithelial malignancy and its relevance to the pathogenesis of oral cancer (part II). Crit Rev Oral Biol Med 15(6):337-347

33. Maeda G, Chiba T, Okazaki M et al (2005) Expression of SIP1 in oral squamous cell carcinomas: implications for E-cadherin expression and tumor progression. Int J Oncol 27(6):1535-1541

34. Pyo SW, Hashimoto M, Kim YS et al (2007) Expression of Ecadherin, P-cadherin and $\mathrm{N}$-cadherin in oral squamous cell carcinoma: correlation with the clinicopathologic features and patient outcome. J Craniomaxillofac Surg 35(1):1-9

35. Lim SC, Zhang S, Ishii G et al (2004) Predictive markers for late cervical metastasis in stage I and II invasive squamous cell carcinoma of the oral tongue. Clin Cancer Res 10(1 Pt 1):166-172

36. Huang Y, Fernandez SV, Goodwin S et al (2007) Epithelial to mesenchymal transition in human breast epithelial cells transformed by 17beta-estradiol. Cancer Res 67(23):11147-11157

37. Guarino M (2007) Epithelial-mesenchymal transition and tumor invasion. Int J Biochem Cell Biol 39(12):2153-2160 